\title{
Meeting Report: AAPS Workshop on Dissolution Testing and Bioequivalence
}

\author{
Vivian A. Gray \\ Dissolution Technologies, Hockessin, DE, USA
}

T he workshop in La Plata, Argentina, was hosted by the National University of La Plata on March 7 and 8, 2013, with all presentations translated into either English or Spanish. All the sessions were followed by a Q\&A panel discussion. The two chairs of the Organizing Committee were Dra. Vivian A. Gray and Dra. Maria Guillermina Volonté. The members of the Organizing committee were Lic. Pablo Quiroga, Msc; Lic. Arturo Hoya; Dr. Raimar Löbenberg; and Dra. Maria Esperanza Ruiz. The Workshop was cosponsored by the AAPS In Vitro Release and Dissolution Testing Focus Group.

Opening remarks were given by Maria Guillermina Volonté from the National University of La Plata followed by four speakers in the morning session. The first talk, given by Vivian Gray from V. A. Gray Consulting, was titled "Method Development and Setting Clinically Relevant Dissolution Specifications, including Quality by Design." Her talk gave principles needed to develop a meaningful dissolution test, one that follows the concepts of QbD. The emphasis was on understanding the product release mechanism and knowing the critical quality attributes, leading to clinically relevant dissolution specifications. The role of variability was discussed, and ways to minimize it were explored. Then María Esperanza Ruiz of the National University of La Plata spoke on "Biopharmaceutical Relevance of the Comparison of Dissolution Profiles." The performance of the main methods proposed for the comparison of percent dissolved versus time curves was analyzed comparatively to propose a more biorelevant combined approach for the comparison of dissolution profiles of multisource drug products. The best correlations were found when the result of the $f_{2}$ similarity factor was combined with a measure of the dissolution extent (e.g., area under the curve). This combined approach gives a robust and informative result with the most biopharmaceutical relevance.

After the break, Arturo Hoya of the National University of La Plata discussed "Impact of API Solid-State Properties on Dissolution and Bioavailability." BCS Class Ila and Ilb are APIs with absorption limited by dissolution rate and solubility, respectively. Reducing API particle size, selecting crystal habit, and increasing solid wettability can enhance the dissolution/absorption of Class Ila APIs. To increase absorption of very low solubility Class IIb APIs, supersaturated drug delivery system (SDDS) are used, in which a high energy solid form (HESF) (metastable polymorph, unstable amorphous API) can dissolve at a high rate and reach high apparent solubility in gastrointestinal fluid (GIF) during enough time to be absorbed. In these cases, an HESF must be formulated as a solid dispersion containing excipients that stabilize the solid state and inhibit or minimize API precipitation in solution. The session ended with Maria Cruanes from Merck giving a presentation on "Biorelevant In Vitro tools to Guide Dissolution Method Development of Low Solubility Compounds: Critical Excipients and Critical API Attributes." She stated that the purpose of in vitro tools is to aid in understanding the mechanism of drug release, screen absorption-enhancing excipients, probe API and formulation attributes, save resources, and speed up development. She described the tool workflow as beginning with in vitro screening, then proceeding to silico simulations, followed by in vivo (animal) studies and lastly in vivo (human) studies. She gave three examples of the efficient use of this tool workflow design.

In the afternoon, Vivian Gray described the American Association of Pharmaceutical Scientists (AAPS), which cosponsored this event. She highlighted the availability of e-membership for Argentinean scientists. The next speaker, Raimar Löbenberg from the University of Alberta, Canada, discussed "Bioequivalence in the Americas Using the WHO Guidelines: An Overview." He began his presentation by defining biowaivers as based on sponsor bioequivalence tests performed with in vitro dissolution instead of a pharmacokinetic study. The method is used in the approval of generics, approval of products at lower doses, or scale-up and post-approval changes (also known as Variations at EMA). He described how the Biopharmaceutics Classification System (BCS) has changed drug regulations. He showed how $B C S$ is interpreted differently by FDA, EMA, JP, and WHO. He also presented several case studies from South America. The problem with insufficient comparator products was discussed, and he stated that global comparator products are needed. He presented the next talk, "Interchangeability of Multisource Products-How to Use In Vitro Methodology," which was prepared by Johannes Krämer of PHAST. This talk began with several definitions such as interchangeability, pharmaceutical equivalence, bioavailability, drug product performance, and bioequivalence. The experimental design of dissolution testing as a QC test and for product comparison was provided. An example of in vitro and in vivo performance was discussed. In vivo and in vitro correlations (IVIVC) were defined and referenced. He went on to show that $\mathrm{BCS}$ is linked to dissolution and absorption factors $D_{n}, D_{0}$, and $A_{n}$. He concluded with the expectations of IVIVC for immediate-release products based on 


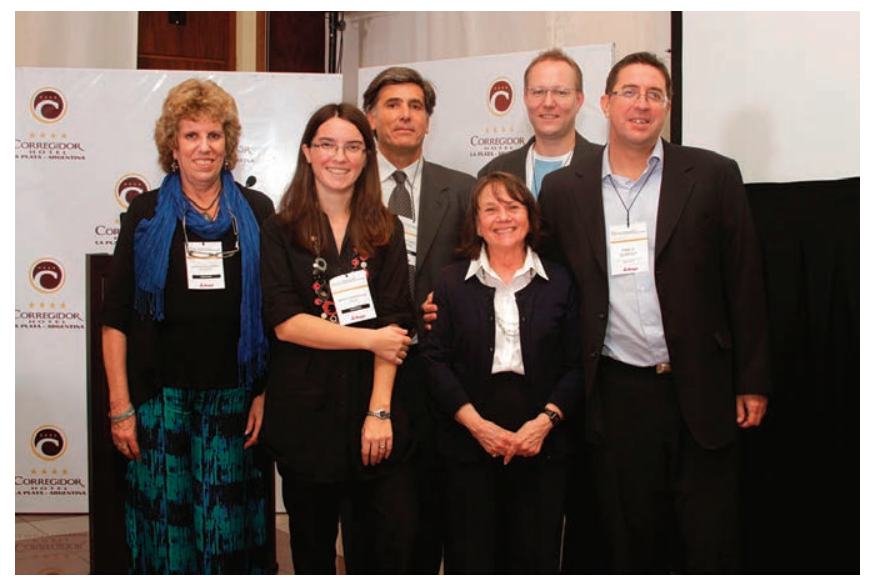

Argentinean Workshop organizers (left to right): Maria Guillermina Volonté, María Esperanza Ruiz, Arturo Hoya, Vivian Gray, Raimar Löbenberg, and Pablo Quiroga.

BCS. Then Raimar Löbenberg continued with a third talk, titled "Computer Simulations to Predict Oral Drug Performance to Establish IVIVC." He began with an overview of $\mathrm{BCS}$, dissolution testing, and IVIVC, and then progressed to the use of dissolution simulations as a predictive tool. More specifically, the in vitro data can be used as the input function into GastroPlus to simulate the absorption profile of drugs. GastroPlus uses a mathematical model called Advanced Compartmental Absorption and Transit (ACAT), which is based on the principles of BCS. The three major input parameters are compound attributes, physiology, and pharmacokinetics. He gave several case studies. He stressed how BCS has changed the way we look at drugs and the drug development process and has made dissolution testing more meaningful. Software can be used to assist in establishing an IVIVC.

On the second day, the session began with Ivana S. Abalos and M. Victoria Mussini from ANMAT speaking on "Bioequivalence Studies in Argentina: Regulatory Framework of the Bioanalytical Stage." From a regulatory point of view, bioequivalence in Argentina is dynamic and adaptable to the improvement of scientific knowledge and health needs. Considering these aspects, a progressive schedule for bioequivalence requirements for certain APIs was established. The analytical stage is highly critical, and it is on these results that the statistical analysis is performed on which the decision of bioequivalence or bioinequivalence between test and reference product is made. It must be ensured that the analytical method used for quantification of biological samples has been properly validated to obtain reliable and consistent results.

The Provision ANMAT N 4844/05 establishes guidelines to achieve quality and reliability of the analytical results of samples from volunteers. It includes the minimum requirements to be met by bioanalytical centers, according to Good Laboratory Practices and technical guidelines for bioanalytical method validation.
The Provision ANMAT N 5040/06 establishes the regime of good practices in the implementation of BE studies. This document refers to the guidelines and requirements for information, as well as the documentation to apply for authorization to conduct a study of BA/BE.

Finally, the Guideline for Good Laboratory Practices in Bioanalytical Centers establishes the requirements to be met by institutions charged for performing the analytical stages of BA/BE studies. They are technically responsible for the truthfulness of the data and information for all processes.

The second speaker, Yanina Rodriguez also from ANMAT, discussed "Standardization of Caco-2 Cell Culture as In Vitro Model for Intestinal Permeability." She introduced the concept of permeability based on the BCS and explained different methods for determining the intestinal permeability of APIs. She explained the Caco-2 cultured system and showed some results obtained in their laboratory. The third speaker was Nikoletta Fotaki from University of Bath, UK. Her topic was "Apparatus 4, Flow-Through Cell, Fundamentals and Applications." She pointed out that in particular, the flow-through cell apparatus is very useful for low solubility drugs, microparticulates, implants, suppositories, and controlledrelease formulations. The key features of the apparatus were then described such as the use of an open system for samples that require a high volume of medium and a closed system for samples requiring a low volume of medium (i.e., $27 \mathrm{~mL} / \mathrm{sample}$ ). The key difference between these configurations is that in using an open system, the raw data are obtained in noncumulative form (amounts dissolved at specific time intervals) instead of in the usual cumulative profiles. She explained that the flow-through cell could also be operated under laminar flow conditions where the sample is situated on a bed of glass beads in the cell column or simply placed in the open column under turbulent flow conditions. She discussed several advantages such as the ability to change medium and flow rate within a single run; efficient simulation of intralumenal hydrodynamics; use of long duration test runs without the problem of evaporation; provision for special dosage forms (floating dosage forms, implants, suppositories); avoidance of carry-over effects; facilitation of IVIVC; and correspondence of a single profile to the release in the entire Gl lumen. However, there are several disadvantages. A high volume of medium is required when used as an open system and additional training is needed compared with the use of standard USP Apparatus 1 or 2, for example. Using the flow-through cell apparatus is labor-intensive, and filtration efficiency may be an issue when using Apparatus 4 with biorelevant media. She then presented several case studies using Apparatus 4.

After the break, Nikoletta Fotaki presented the next talk, "IVIVC According to USP General Chapter <1088> In Vitro and In Vivo Evaluation of Dosage Forms," which was prepared by Johannes Krämer. Through practi- 
cal examples, his talk illustrated ways of applying USP $<1088>$, performing IVIVC level A and B correlations, gathering additional information from correlation plots, and determining reliability in an IVIVC. In the description of experience with Level B correlations, statistical moment theory, the mean times concept was presented. The goals of a dissolution test should consider the critical quality attributes, evaluating the robustness as a parameter of the drug product and a prediction of bioavailability. Then Carlos Scoccia from Lab. Bagó - Argentina gave a presentation on "Dissolution Profile as a Tool of Pharmaceutical Product Development." The aim of this lecture was to highlight the importance of the dissolution profile on the pharmaceutical development process, with a particular focus on the pharmaceutical dosage form. He used a conceptual map to describe the relationship between the elements and underlying factors that are part of the dissolution profile in vivo. It is necessary to develop dissolution methods with biological significance and to try to predict the in vivo behavior of a pharmaceutical dosage form. This tool provides regular feedback of information during the entire pharmaceutical development process to select the optimal physicochemical and mechanical properties of the drug, determine both suitable excipients for the formulation and process variables for the manufacture of the dosage form (operative space design), and understand the kinds of post-approval changes that can be performed on the product.

The last session of the workshop started with Maria Cruanes, who discussed "When and How to Implement Quality by Design (ICH Q8): An Industry Perspective." Merck has embraced the QbD concept. QbD starts at API selection and continues during $R \& D$, scale up, and finally production. QbD is implemented by starting with patient needs following ICH Q8, Q9, and Q10 guidances; understanding the science and technology; assessing risk management; and clearly defining the work process. Nikoletta Fotaki then returned to present her topic "From Dissolution to BA/BE Studies, Including a European Perspective." She gave illustrations of where modeling and appropriate in vivo data led to successful development of IVIVCs. There is a new draft of a European (EMA) guidance that now thoroughly discusses IVIVC. She described the draft guideline in depth. The last speaker was Pietro Fagiolino from the University of the Republic (Uruguay). His presentation was "New Clinical Approaches to the Bioequivalence Study." Mean drug concentration profiles do not account for the inter- and intra-individual variability of the test/reference (T/R) drug-exposure ratio, and hence different subpopulations could present opposite $T / R$ ratios, or the same individual could display different $T / R$ ratios under two different (time/condition) drug administration protocols (day-night/fed-fasting), among others. Then, interchangeability of bioequivalent products becomes controversial for all patients regardless of the mode of drug product administration. His research

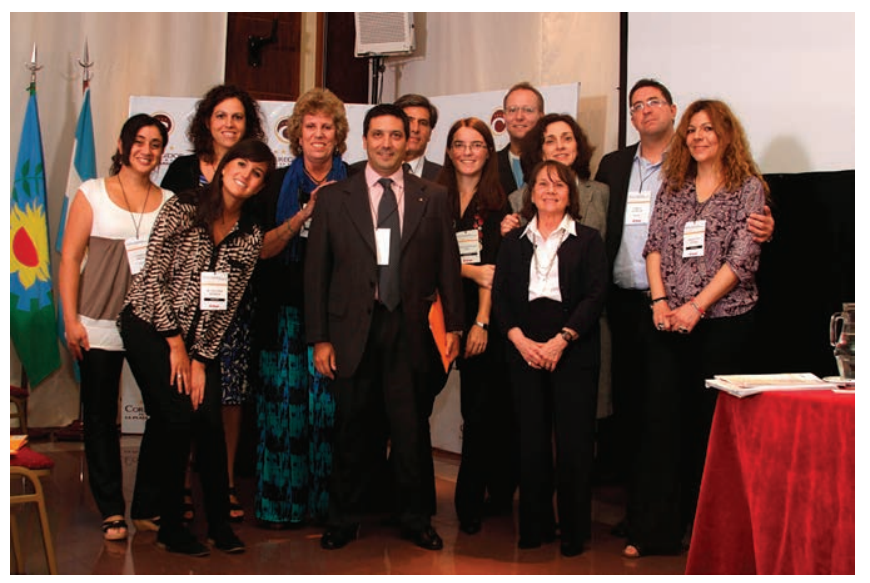

Argentinean Workshop speakers and moderators: Ivana S. Abalos, Yanina Rodriguez, M. Victoria Mussini, Maria Guillermina Volonté, Carlos Scoccia, Arturo Hoya, María Esperanza Ruiz, Raimar Löbenberg, Maria Cruanes, Vivian Gray, Pablo Quiroga, Nikoletta Fotaki, and Pietro Fagiolino (not pictured).

group at the Bioavailability and Bioequivalence University Center for Medicines Evaluation (Uruguay) has found different T/R drug plasma concentration ratios when women and men or when fasting and coprandial administration were analyzed. The main reason why two pharmaceutical products were dissimilarly discriminated seems to lie in the gastric residence time. In women and under coprandial administration, there is a delay in gastric emptying, and thus the drug has more time to reach the intestinal absorption site. In men and under fasting administration, rapid gastric emptying does not give enough time for a drug that is delivered at different rates from test and reference dosage to enter the intestine equally. To give accurate information about the best clinical setting to assure both effective and safe interchangeability between bioequivalent drug products, he proposed to extend bioequivalence research assaying in different subpopulations (sex-, age-, genotype-related) and under different administration setting (day-night, fed-fasting, winter-summer, resting-physically active, etc.). Current bioequivalence assessments are rigorous enough to give commercial authorization; however, beyond therapeutic equivalence, biopharmaceutical equivalence should be precluded when two drug products containing the same active ingredient are declared bioequivalent.

Vivian Gray closed the workshop with remarks of thanks to the Argentinean organizers and speakers and appreciation for the fine hospitality shown to the AAPS Focus Group speakers. She remarked on the high quality of speakers and audience participation and thanked the translators and organizers for an excellent job.

There were 133 attendees from eight countries: Argentina, Brazil, Canada, Chile, Paraguay, Uruguay, United Kingdom, and United States. There were many student attendees with nine universities in Argentina represented. 